\title{
Architecture for Discovery and Customization of Multi-tenant Learning Process as a service and resources allocation in cloud computing
}

\author{
Sameh Azouzi \\ ISITCom Hammam Sousse \\ Laboratory RIADI-GDL, ENSI, \\ Mannouba, Tunisia \\ Azouzissameh@yahoo.fr
}

\author{
Sonia Ayachi Ghannouchi \\ ISG Sousse/ Laboratory RIADI-GDL, \\ ENSI, Mannouba, Tunisia \\ s.ayachi@coselearn.org
}

\author{
Zaki Brahmi \\ ISITCom Hammam Sousse \\ Laboratory RIADI-GDL, ENSI, \\ Mannouba, Tunisia \\ zakibrahmi@gmail.com
}

\begin{abstract}
Collaborative e-learning based on several means of electronic communication mechanism specifically internet and web2.0 (such as forum, virtual classroom, videoconference, etc.) has become the current trend in e-learning systems. The cloud computing environment presents itself an important infrastructure for supporting these collaborative e-learning systems and for optimizing these service-based systems in a multi-tenant way. In this paper, we discuss the problem of discovering multi-tenant e-learning processes. For this, we propose an architecture for a configurable learning process discovery environment and the allocation of resources (Saas, Paas, Iaas) necessary for the execution of the corresponding learning activities.
\end{abstract}

Keywords- Discovery e-learning process; collaboratif elearning; business process; resource allocation; cloud computing.

\section{INTRODUCTION}

E-learning is defined as Internet-enabled learning [1]. ELearning components can include multi-format content, knowledge sharing, inclusion of collaboration tools, and social networking. It is important to emphasize the value of collaboration and knowledge sharing between learners and teachers in e-learning systems. In this context, great attention is given by researchers, developers and educators to collaborative learning through Learning Management Systems (LMS). However, several studies in literature show some limitations of these e-learning platforms (LMS) [4] [5] [6]. These limitations are mainly at the level of collaboration and interaction between different actors of an e-learning activity; they based mainly on texts and photos. The form and effect of collaboration and interaction are relatively simple and limited.

DOI reference number: 10.18293/SEKE2019-136
So, it is unrealistic for each school to offer its own elearning system that responds to learner expectations and manages the new neo-digital society. In order to remedy these different LMS deficiencies, we have contributed in previous work [7] to a general, collaborative, configurable and multi-tenant e-learning system. The collaboration we propose is synchronous and asynchronous. And we also suggest using the business process line approach (BPL) inspired by the Software Product Line (SPL) approach for the development of multi-tenant e-learning applications. The proposed general e-learning process could be implemented in a variety of contexts and accommodates the different needs of universities and teachers. However, all variants of our model share a set of common elements / activities and others differ by some variable activities.

Thus, the adoption of an SPL and BPL approach in the field of e-learning seems to be a promising solution. On the one hand, it overcomes the limitations of LMS systems, and on the other hand, it provides institutions with e-learning applications tailored to their needs.

Motivated by the difference and diversity of the needs of tenants (teachers / learners), institutions are looking for elearning systems available outside their organizations that are rapidly adapting to the new demands of their teachers and learners and reducing costs of development and maintenance. Cloud computing is the best solution because of its ability to outsource service-based learning processes with a pay-per-use model.

According to the National Institute of Standards and Technology (NSIT), cloud computing is a model that allows providers to share their resources (services, networks, storage servers, and applications) and the users who access them in a ubiquitous, and convenient way of request [8]. In a multi-tenant environment, the use of a configurable learning process provides configurable learning that can be configured by different tenants based on their specific needs 
[9]. Different approaches for modeling multi-tenant configurable processes have been proposed; mainly they focused on configurable business process discovery and focused on configurable resource allocation. Thus, in this sense, we propose a discovery architecture of learning services considered as business processes in the cloud. In our architecture, we also offer discovery of other Saas, Paas, Iaas cloud services and collaborative web2.0 services that facilitate the deployment and execution of all types of learning activities in different contexts. But some activities require a wide bandwidth or a very powerful CPU; other activities require collaborative web2.0 applications and SaaS services to be executed.

In order to overview all these aspects, this contribution is arranged as follows.

In Section 2 we introduce the main concepts of Cloud Computing, e-learning including its infrastructure and main layers, configurable learning process, multi-tenant and resource allocation.

Next, Section 3 describes our proposed architecture. Finally, the main concluding remarks are given in Section 4.

\section{PRELIMINAIRES}

In this section, we give a brief description of related topics, which are utilized in this work.

\section{A. Configurable learning process}

The world of education and training has embraced new methods and new pedagogical tools. Since the computer, at first, and the Internet, then, have appears in classrooms, departments but also and especially in the daily life of each of the actors involved, the school (in the most generic sense of the term) is engaged in a real race to stay the more possible in line with the reality of society. Each school faces a major challenge, which is the development of a learning system that manages IT innovations and meets the needs of teachers / learners knowing that they do not cease to evolve. Starting from this point, there comes the idea of developing a configurable e-learning process. A configurable process model allows the sharing of a process between different tenants, which can be customized according to specific needs. To model a configurable learning process, one must study the variability of this process. In our work, we view the e-learning process as a multi-tenant business process and each tenant can choose a sub-process (configuration) of the overall process. With respect to business process variability, tenants can have varying business workflows. Therefore, the application must allow configuration and customization to meet the tenant's goals and requirements [23]. In previous work we have presented the modeling of a multi-tenant configurable general e-learning process [7,24].

\section{B. Cloud computing and e-learning}

Cloud computing uses a new technology that develops applications in a way that allows both the execution of the application and the storage of data to be performed ubiquitously for all users. Cloud architecture provides user support at different levels that vary according to their different characteristics. The different levels can be described as follows [3]:

- Infrastructure as a Service (IaaS): remote management and control of hardware resources provided by a system.

- Platform as a Service (PaaS): offers the cloud platform along with a series of libraries to develop applications in which the distribution of tasks, the persistence and other layers are transparent for the developer.

- Software as a Service (SaaS): consists in offering different applications to be used through the internet as opposed to a local installation.

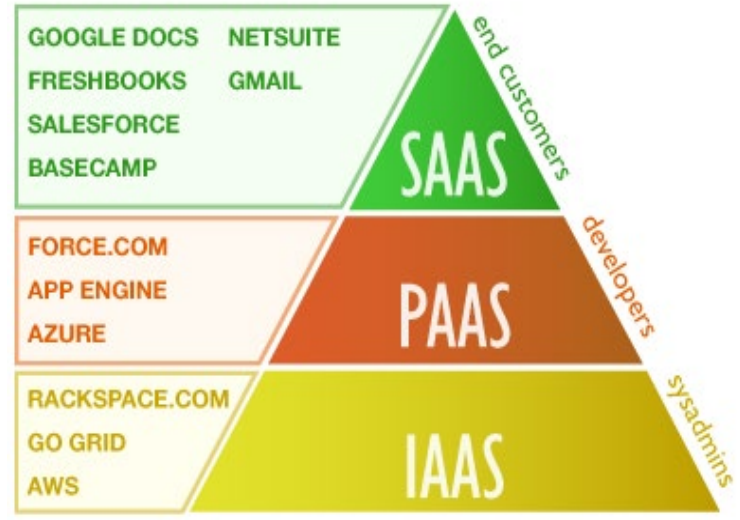

Figure 1. Illustration of the layers for the Services Oriented Architecture [12]

There are incipient developments that broach the topic of cloud technology and e-learning [2], however few studies incorporate both concepts. The success of the solution software as a service (Saas) is real and is easily applicable for e-learning. Cloud computing, Saas and e-learning are completely complementary. Accordingly, we note that the focus on changing traditional e-learning systems to more collaborative interactive learning environments has increased. J. Méndez \& J. Gonzalez [3] highlights limitations of traditional e-learning pointing that system creation and maintenance are placed inside the educational institutions, which can cause a lot of problems including huge investments with no gain and lack of development potential. In 2009, cloud computing is presented by [20] as a new computing model to implement e-learning ecosystem to overcome the problems in the traditional system. The author considers that cloud computing is able to add some critical features to e-learning ecosystem, such as: configuration at real-time, utilization of resources, on demand resource sharing and better management for software or hardware [20]. The cloud-based system supports the construction of a new generation of e-learning systems that is accessible from a wide range of hardware devices, while storing data inside the cloud.

B. Dong \&al., [22] proposes a platform architecture based on the integration of cloud computing and web 2.0 for developing intelligent virtual learning community and makes the learning environment more productive, scalable, flexible and adjustable towards students demands and needed 
information and communication technologies. The author states that the usage of cloud computing and web2.0 for elearning affects the way e-learning software projects are managed and the proposed intelligent virtual learning community enhances the efficiency of learning environment, provides up-to-date resources, constancy, guaranteed quality of service, dependability, scalability, minimized time, efficient usage of resources, flexibility, and maintaining of elearning system.

Within the same context, Ouf, Nasr and Helmy [21] have proposed an e-learning system based on the integration of cloud computing and Web 2.0 technologies to meet the requirements for e-learning environment such as flexibility and compliance towards students' needs and concerns, improve and enhance the efficiency of learning environment.

Aljenaa \& al., in [23] introduced the main components of a system of effective e-learning through using cloud computing technology. The authors insist on the importance of the following components:

- "Cloud software platform" which contains the LMS platform and the necessary tools of collaboration and communication to meet the needs of learners throughout the process of learning;

- $\quad$ "Operational and management components" to make the management of the learning process;

\section{Multi-tenancy}

Multi-tenancy being one of the key features of service in cloud, the service providers can offer single application to multiple users. A multi-tenant application caters a personalized customer experience improving performance and efficiency and provides a flexible space for customers to efficiently pursue new business demands. From the software vendor perspective, such multi-tenant applications, improve resource utilization and reduce the operational costs in delivering the software as a service. Each of the tenants may have unique requirements that differ from each other. Customization plays a vital role to converge specific requirements of every tenant. It is up to the vendor, what extent of customization to be offered to the tenant [10]. Two main goals of multi-tenancy are isolation and sharing, which are contradictory [11]. As we discussed in the introduction, the primary motivation of multi-tenancy is to enable management of the needs of universities/learners that are rapidly changing and diversified. However, at the same time, each user needs complete isolation and often does not want to know even the existence of the other users. Furthermore, a multi-tenant setup will inevitably attract more requests, and therefore must be scalable. Scalability in a multi-tenant setup has several dimensions: that is supporting large number of requests, processes, and tenants [11].

\section{Resource allocation in cloud-based Business process}

In previous work, we considered e-learning processes as business processes, because of the similarity between the two in the orchestration of learning activities and that of business processes [7]. Thus, we use the BPFM approach for modeling configurable e-learning processes in the cloud. Running a learning process requires Iaas-level resources that can be distributed across virtual machines (VMs), SaaS services and applications, and Paas platforms. Until today, research on business process discovery in the cloud and resource allocation has been rare. There are works such as $[13,14]$, which focus on the allocation of human resources. Some other works explicitly consider the characteristics of the cloud: In [18], the authors proposed an approach for configurable cloud resources allocation in multi-tenant business process. Their aim is to shift the cloud resource allocation from the tenant side to the cloud process provider side for a centralized resource allocation management. Through configuration, different tenants can easily customize the selection of the needed resources taking into account two important properties: elasticity and shareability.

S. Schulte \& al. In $[15,16]$ develop a Platform for Elastic Processes, which combines the functionalities of a BPMS with that of a Cloud resource management system. Recently, work in the area of configurable process modeling has been proposed with perspectives for resource allocation. La Rosa $\&$ al. In [25] Offer C-EPC (configurable event driven process chains) with resource, data and physical object capture capabilities. However, they focus on human resources and there is no support for cloud resources. In [17], A. Hallerbach \& al. Extend process variants with options (Provop) to properly model and manage large collections of process variants. Juhnke $\&$ al. [18] provide an extension to a standard BPEL workflow engine, which allows the use of Cloud resources to execute business processes. The same applies to the work by Bessai \& al. [19], who also assumes that workflows are composed of single software services. The authors offer different methods to optimize resource allocation and scheduling, pareto-optimal solution covering both cost and time. E. Hachicha \& al. [26] proposed an approach for configurable cloud resource allocation in multitenant business processes and to shift the cloud resource allocation from the tenant side to the cloud process provider side for a centralized resource allocation management. Table I summarizes these approaches and relates them to properties that are important in a cloud setting. We observe that resource variability, cloud features, and allocation are only partially covered or not at all. In the following, we aim to fill these gaps by the definition of our novel approach which allows the configurable e-learning process discovery and allows the cloud resources allocation needed for the learning activities execution such as IaaS, PaaS and SaaS. 
TABLE I. Evaluation of previous approaches

\begin{tabular}{|c|c|c|c|c|c|c|c|}
\hline $\begin{array}{c}\text { Criteria } \\
\text { Approack }\end{array}$ & $\begin{array}{c}\text { Control- } \\
\text { flow } \\
\text { variability }\end{array}$ & $\begin{array}{c}\text { Multy- } \\
\text { tenancy }\end{array}$ & $\begin{array}{c}\text { Ressource } \\
\text { variability }\end{array}$ & $\begin{array}{c}\text { Ressource } \\
\text { allocation } \\
\text { Saas }\end{array}$ & $\begin{array}{c}\text { Ressource } \\
\text { allocation } \\
\text { Iaas }\end{array}$ & $\begin{array}{c}\text { Ressource } \\
\text { allocation } \\
\text { Paas }\end{array}$ & $\begin{array}{l}\text { BP- } \\
\text { discovery in } \\
\text { cloud }\end{array}$ \\
\hline$[18]$ & - & + & - & - & + & - & - \\
\hline$[15,16]$ & + & - & - & - & + & - & - \\
\hline$[17]$ & + & + & - & - & - & - & - \\
\hline$[19]$ & - & - & - & - & + & - & - \\
\hline$[26]$ & + & + & + & - & + & - & - \\
\hline Our approach & + & + & + & + & + & + & + \\
\hline
\end{tabular}

\section{E. Discussion}

We can conclude by considering that all these research works concerning the systems of e-learning in the cloud, integrating the multi-tenant aspect and allocating the resources needed to execute an e-learning process suffer from some limits namely:

The absence of a complete initiative or approach for the construction of an agile and reconfigurable process of learning adaptable to change and being able to evolve to meet the needs of learners;

- Lack of collaboration in learning processes and limited re-use possibilities from the processes deployed by other universities/teachers;

- The allocation of resources in the cloud is limited to the infrastructure level.

In our research work, we considered an e-learning process as a multi-tenant configurable business process in the cloud (BPaas) and to achieve this goal we combined the Software Product Line (SPL) and Business Process Management approach (BPM).

In the next section we will:

- Provide an approach for discovering a multi-tenant configurable e-learning process and configurable cloud resource allocation.

- The allocation of cloud resources (Iaas, Paas, Saas) configurable in multi-tenant e-learning process.

\section{MULTI-TENANCY AWARE CONFIGURABLE LEARNING PROCESS AS A SERVIE DISCOVERY ARCHITECTURE}

The goal of this research work is the development of multi-tenant, configurable and collaborative learning process, that utilizes BPM and SPL for modeling the learning process and cloud computing. In cloud computing environments, there are two actors: the providers and the cloud computing users. On the one hand, suppliers hold massive resources in their large data centers and rent these resources to users. On the other hand, there are users who have applications with various loads and rent resources from the providers for running their applications. Once the final deployment solution has been defined and the learning process is deployed, multi-tenant configurable learning process description are created and published by learning process providers into service registries hosted in cloud data centers. In the datacenter registers, there are also descriptions of other cloud services namely Saas, Paas, Iaas and others collaborative Web2.0 services that have been used in the execution of the process. Published learning process as a services artifact are specified as business processes. The proposed discovery architecture provides personalized learning process configurations for tenants as presented in figure 2. 


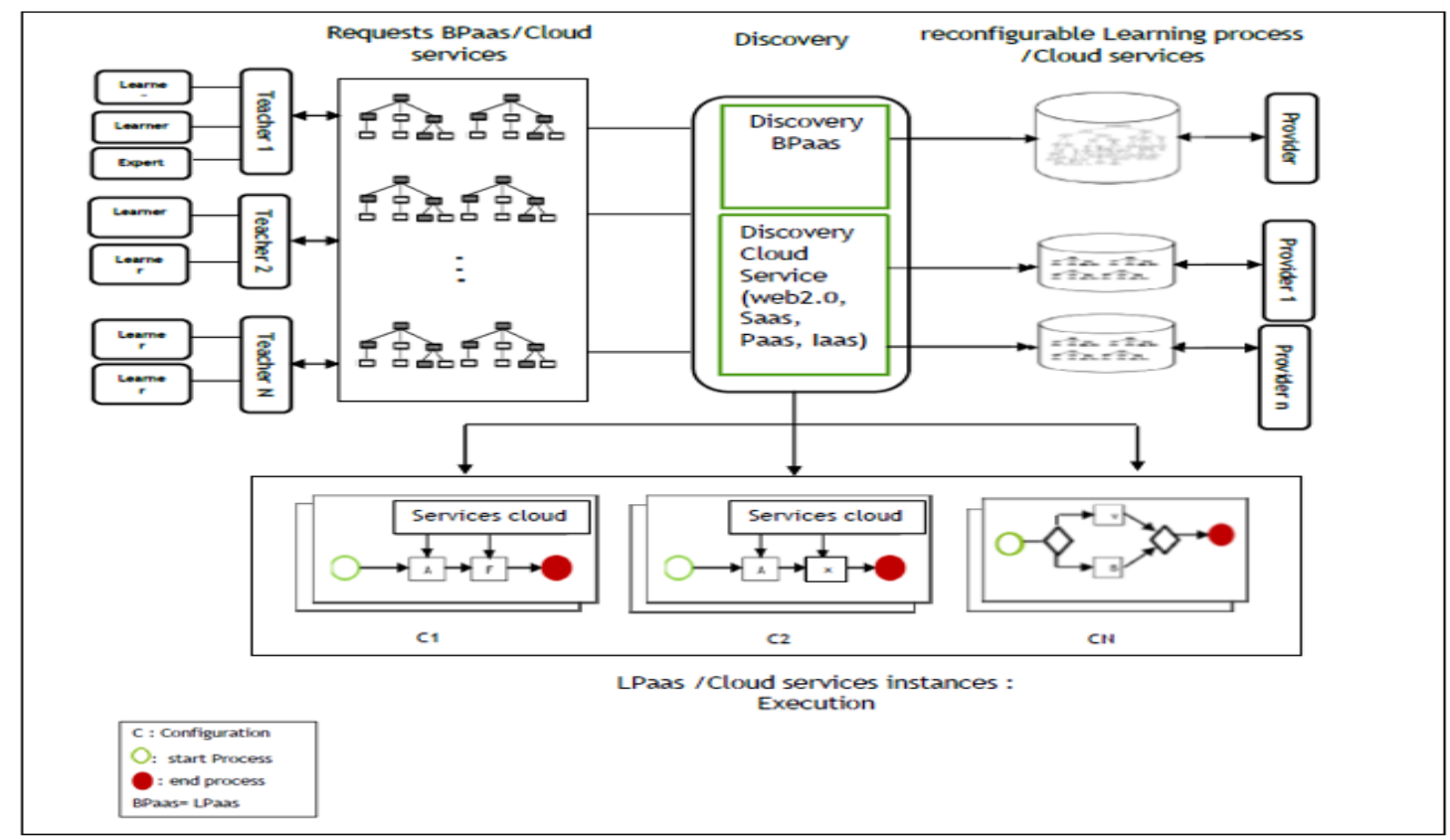

Figure 2. Conceptual architecture of Multi-tenant aware configurable learning process as a service discovery architecture and allocation of cloud ressources

The discovery system consists of a modular architecture organized into separate modules: Request BPaas/ Cloud services, Discovery, Reconfigurable learning process/ Cloud services, LPaas/ Cloud services instances (execution).

Each module is composed of one or more components cited as follows:

- Reconfigurable learning process / cloud services: Our configurable learning process is created and published in configurable service registries hosted in cloud data centers. Thus, cloud services such as Saas, Paas, Iaas and collaborative web2.0 services are published by several cloud service providers. These services will be used in the execution of the elearning process. This is what the allocation of resources required for the execution of such activity requires.

- Requests BPaas/ Cloud services: Tenants who are in our case Institutions / Teachers look in the registers for configurable e-learning process configurations. They can formulate their required process configuration by selecting a set of activities. So, they look for cloud services that they want to use in running selected activities.

- Discovery: this module consists of two sub-modules: BPaas discovery and cloud services discovery. The "BPaas Discovery" module run to locate, discover and generate the appropriate configuration. This module uses another cloud service discovery module "discovery cloud service" to discover collaborative web2.0 services and other services (Saas, Paas, Iaas). These services will be used to carry out the selected activities, collaborate with experts, share knowledge and pedagogical resources, and strengthen the infrastructure to carry out such a task.
- LPaas / cloud services instances (runtime): The return of the discovery module are instances of BPaas / cloud services that respond to a request configuration. Once the recommended configuration is generated, it will be run as a business process in the cloud.

The multi-tenant aware discovery of configurable business process as a service is one of the most important and difficult issues, because of multiplicity and nonstandardization of their description in cloud. In this paper, we introduce the combination of BPM approach and SPL approach for modeling collaborative, configurable e-learning process; then we introduce our architecture for discovery of the learning process in cloud. Our approach empowers multiple tenants to discover their desired learning process service configured variants, considering individual variants. To do so, we reduce the problem of configurable matching to a tree matching problem and we are going to adapt existing algorithms for this aim. We are doing the necessary experiments to show the feasibility of our approach.

\section{CONCLUSION}

In this work, we exposed the limitations of existing elearning systems (LMS) and discussed our contribution in previous work on modeling a collaborative, configurable and multi-tenant e-learning process. with the use of the Business Process Feature Model (BPFM) approach. Subsequently, we exposed the basic concepts of deploying a learning process as a business process (BPaas) in the cloud: the different layers of cloud computing, the multi-tenant aspect and the supply of resources for running a process in the cloud.

For this, we propose an architecture of a discovery framework of a learning process and the allocation of resources cloud computing which allows the personalization 
of e- learning services and the selection of collaborative web 2.0 services, services to infrastructure level and platform level.

In our future work, we are going to engage in showing the feasibility of our architecture where we rely on the structural matching approach and the graph-edit distance for e-learning BPaas discovery in the cloud.

\section{REFERENCES}

[1] Mayer, R., Clark, R.: E-Learning and the Science of Instruction: Proven Guidelines forConsumers and Designers of Multimedia Learning, 3rd edn. Pfeiffer (2011).

[2] Ercan, T.: Effective use of cloud computing in educational institutions. Procedia Socialand Behavioral Sciences 2, 938-942 (2010).

[3] J. Méndez, J. Gonzalez, "Implementing motivational features in reactive blended learning : Application to an introductory control engineering course", Education, IEEE transaction, vol. 54, no. 4, pp. 619-627, 2011

[4] V. Stantchev, R. Colomo-Palacios, P. Soto-Acosta, and S. Misra, "Learning management systems and cloud file hosting services: A study on students' acceptance," Computers in Human Behavior, Vol. 31, February 2014, pp. 612-619.

[5] M. A. Conde, F. García, M. J. Rodríguez-Conde, M. Alier, and A. García-Holgado, "Perceived openness of Learning Management Systems by students and teachers in education and technology courses,"Computers in Human Behavior, Vol. 31, February 2014, pp. $517-526$

[6] Z. Du, X. Fu, C. Zhao, Q. Liu, and T. Liu, "Interactive and Collaborative E-Learning Platform with Integrated Social Software and Learning Management System,"Proceedings of the 2012 International Conference onInformation Technology and Software Engineering, Lecture Notes in Electrical Engineering, Vol. 212, 2013, pp 11-18.

[7] Sameh Azouzi, Sonia AyachiGhannouchi, Zaki Brahmi: "Modeling of a Collaborative Learning Process with Business Process Model Notation", International Conference on Digital Economy, pp. 95104, 2017.

[8] Mell, Peter, and Tim Grance. "The NIST definition of cloud computing." (2011).

[9] Aalst, W.: Business Process Con guration in The Cloud: How to Support andAnalyze Multi-Tenant Processes? In: ECOWS, IEEE (2011) 3-10.

[10] Ankit Bhilwar, Sandesh Jain, others: "Multi-tenant enabled eLearning platform: Blended with workflowtechnologies", e-Learning, e-Management and e-Services (IC3e), 2014 IEEE Conference on, pp. $88-92,2014$.

[11] MilindaPathirage, Srinath Perera, IndikaKumara, SanjivaWeerawarana: "A multi-tenant architecture for business process executions", Web services (icws), 2011 ieee international conference on, pp. 121-128, 2011.

[12] Fernandez, A, Peralta, D, Herrera, F., et al. An overview of elearning in cloud computing. In : Workshop on Learning Technology for Education in Cloud (LTEC'12). Springer, Berlin, Heidelberg, 2012. p. 35-46.

[13] Cabanillas, C., et al.: Towards process-aware cross-organizational human resource management. In: BPMDS, EMMSAD CAiSE, Greece, June 16-17. (2014) 79-93.

[14] Kajan, E., et al.: The network-based business process. IEEE IC 18 (2014) 63-69.

[15] Schulte, S., et al.: Costdriven optimization of cloud resource allocation for elastic processes. In: International Journal of Cloud Computing. (2013) 2326-7550.

[16] Schulte, S., et al.: Realizing elastic processes with viepep. (In: Service-Oriented Computing - ICSOC, China, Nov 12-15, 2012, Revised Selected Papers) 439-442.

[17] Hallerbach, A., et al.: Capturing variability in business process models: The provop approach. Journal of Software Maintenance and Evolution: R \& P (2010) 519-546.

[18] Juhnke, E., Dörnemann, T., Bock, D., Freisleben, B. (2011). Multiobjetive Scheduling of BPEL Workflows in Geographically Distributed Clouds, Proc. of IEEE 4th Intern. Conf. on Cloud Computing (CLOUD 2011), Washington DC, USA, 412-419.

[19] Bessai, K., Youcef, S., Oulamara, A., Godart, C. (2013). Bi-criteria Strategies for Business Processes Scheduling in Cloud Environments with Fairness Metrics, Proc. of IEEE 7th Intern. Conf. on Research Challenges inInformation Science (RCIS 2013), Paris, France, 1-10.

[20] D. Chandran, "Hybrid E-learning platform based on cloud architecture model: A proposal", in Signal and Image Processing (ICSIP), 2010 International Conference on, Chennai, 2010, pp. 534 537.

[21] S. Ouf, M. Nasr, and Y. Helmy, "An enhanced e-learning ecosystem based on an integration between cloud computing and Web2.0"? in Signal Processing and Information Technology (ISSPIT), 2010, pp. $48-55$.

[22] B. Dong, Q. Zheng, J. Yang, H. Li, M. Qiao, "An e-learning ecosystem based on cloud computing infrastructure", in Advanced Learning Technologies, 2009. ICALT 2009. Ninth IEEE International Conference, Riga, 2009, pp. 2009125 - 127.

[23] AssylbekJumagaliyev, Jonathan Nicholas David Whittle, Yehia Said Shahat Ahmed Elkhatib: "Evolving multi-tenant SaaS cloud applications using model-driven engineering", 2016.

[24] Azouzi Sameh, Zaki Brahmi, and Sonia Ayachi Ghannouchi. "Customization of multi-tenant learning process as a service with Business Process Feature Model." Procedia Computer Science 126 (2018): 606-615.

[25] Rosa, M.L., et al.: Configurable multi-perspective business process models. Inf. Syst. 36 (2011) 313-340

[26] Hachicha, Emna, et al. "A configurable resource allocation for multitenant process development in the cloud." International Conference on Advanced Information Systems Engineering. Springer, Cham, 2016. 